ANNALES

UNIVERSITATIS MARIAE CURIE-SKŁODOWSKA

LUBLIN - POLONIA

VOL. LXX, 1

SECTIO AA

2015

\title{
Wetting properties of the sodium bis(2-ethylhexyl) sulfosuccinate (AOT) and polyoxyethylene (23) lauryl ether (Brij 35) binary mixtures in the poly(tetrafluoroethylene)-solution-air system
}

\author{
Joanna Krawczyk \\ Department of Interfacial Phenomena, Faculty of Chemistry, \\ Maria Curie-Skłodowska University, \\ Maria Curie-Skłodowska Sq. 3, 20-031 Lublin, Poland \\ e-mail: j.krawczyk@poczta.umcs.lublin.pl
}

\begin{abstract}
Wettability of poly(tetrafluoroethylene) (PTFE) by aqueous solutions of binary mixtures composed of sodium bis(2-ethylhexyl) sulfosuccinate (AOT) with polyoxyethylene (23) lauryl ether (Brij 35 ) was considered on the basis of the measured values of contact angle and surface tension. It was shown that the value of the critical surface tension of PTFE surface wetting at the studied system $(23.5 \mathrm{mN} / \mathrm{m})$, does not depend on the concentration and composition of the binary mixtures of studied surfactants in water, and it was higer than the surface tension of PTFE $(20.2 \mathrm{mN} / \mathrm{m})$. The best wettability of polytetrafluoroethylene (PTFE) by studied aqueous solutions of binary surfactants mixtures occurs at the mixtures concentration corresponding to the critical micelle concentration of their solutions.
\end{abstract}

Keywords: surfactant, polymer, contact angle, critical surface tension of wetting.

\section{INTRODUCTION}

Wetting of solids by surface active agents is important for many technological applications, such as oil recovery, coating, adhesion, 
flotation, printing, detergency and cosmetics industry [1]. Surfactants play an important role in many processes of interest in both fundamental and applied science. Because of their hydrophilic-hydrophobic structure they are able to adsorb at the interfaces and to form colloidal-sized clusters in solutions, known as micelles. The most popular solutions are those based on water. The surface active agents adsorb at water-air and solid-air interfaces reducing the water surface tension $\left(\gamma_{L V}\right)$ and solid-water $\left(\gamma_{S L}\right)$ interface tension to values which cause contact angle $(\theta)$ decrease in hydrophobic low energetic solid-solution drop-air systems. In the case of polar solid-solution drop-air systems surfactant adsorption at solid-water interface does not always reduce the solid-water interfacial tension [2]. Because of the fact, the contact angle changes are sometimes difficult to predict. The influence of surfactants on the contact angle in solid-waterair systems was studied by many investigators [2-6] and in different systems, also those including natural surfaces [3, 7-10]. (PTFE)

The solid-liquid interface tension can, among other things, be determined from the Young equation by measuring the contact angle $[2,11]$ :

$$
\gamma_{L V} \cos \theta=\gamma_{S V}-\gamma_{S L}
$$

where $\gamma_{S V}$ is the solid surface tension.

Equation (1) and Gibbs equation [2] were taken into account by Lucassen-Reynolds [12] to analyze the adsorption of a surfactant at solidair, liquid-air and solid-liquid interfaces:

$$
\frac{d\left(\gamma_{L V} \cos \theta\right)}{d \gamma_{L V}}=\frac{\Gamma_{S V}-\Gamma_{S L}}{\Gamma_{L V}}
$$

where $\Gamma_{S L}, \Gamma_{L V}$ and $\Gamma_{S V}$ are the surface excess concentration of the surface active agents at the solid-solution, solution-air and solid-air interfaces, respectively and $\gamma_{L V} \cos \theta$ is the adhesion tension. Bernett and Zisman [13,14] showed a straight linear relationship between $\cos \theta$ and the surface tension of liquids or aqueous surfactant solutions. The extrapolation of this relationship to $\cos \theta=1$ allows us to estimate the liquid surface tension required to give the contact angle of zero degree, which Zisman [15] described as the critical surface tension of wetting $\left(\gamma_{C}\right)$. But Bergman and van Voorst Vader [16] showed a straight linear 
relationship between the adhesion tension and surface tension of aqueous solutions of several types of surfactants.

From equation (2) it results that it is possible to determine the ratio of $\left(\Gamma_{S V}-\Gamma_{S L}\right)$ to $\Gamma_{L V}$. However, if for the aqueous solution of a given surface active agents in the total range of their concentration $\Gamma_{S V}$ is constant then it is possible to determine $\Gamma_{S L}$ knowing $\Gamma_{L V}$.Using the equation (2) it was found, that for completely nonpolar surfaces, for example PTFE, the $\Gamma_{S L}$ and $\Gamma_{L V}$ were the same because the slope of the line representing the relationship between $\gamma_{L V} \cos \theta$ and $\gamma_{L V}$ was equal -1 . It indicates that adsorption at solid-liquid and liquid-air interface is the same [2]. In the case of polar solids the slope of $\gamma_{L V} \cos \theta$ versus $\gamma_{L V}$ curve differs significantly from $-1[2,17]$. It results from the fact that adsorption of surfactants at solid-liquid interface is different than at liquid-air interface.

Many investigators [13-16, 18, 19] suggest that the liquid whose surface tension value is equal or lower than that of solid should wet it. It means that decrease of the water surface tension to that of solid by the addition of the surface active agent to water should give the spontaneous spreading of aqueous solution of surface active agents over the solid surface. On the other hand, according to the thermodynamic rules such condition is fulfilled if the solid-solution interface tension is equal to zero and when the surface tension of solution and solid is the same [2]. However, as follows from for example $[20,21]$ in the case of aqueous solutions of surfactants the critical surface tension of solid wetting is somewhat higher or considerably lower than the solid surface tension and in many cases it depends on the kind of surfactant added to water. Such behaviour of aqueous solution of surfactants in the wetting process is caused on one hand by the changes of the solid-solution interface tension, which strongly depends on the orientation of surfactant molecules in the surface layer at the solid-solution interface different from that at the solution-air interface. Thus, the changes of the solid-solution interface tension can occur in different direction from that of water surface tension. Usually, the addition of the single surfactant to water does not guarantee the possibility to wet spontaneously a given solid, because of that mixtures of surfactants are very often applied [1]. In the case of the mixture of surfactants not only the changes of water surface tension can be different from that of a single one but also the solid-water interface tension. In the literature, the problem dealing with different influence of 
both single and mixtures of surfactants on the solid-water interface tension as a function of the total surfactants concentration is not fully explained. Thus, the purpose of the paper was to study the influence of the binary mixtures of anionic and nonionic surfactants on the poly(tetrafluoroethylene) (PTFE) surface wetting. For this purpose the contact angle of the aqueous solution of sodium bis(2-ethylhexyl) sulfosuccinate (AOT) and polyoxyethylene (23) lauryl ether (Brij 35) mixtures on the PTFE surface was measured.

\section{EXPERIMENTAL}

\subsection{Materials}

Sodium bis(2-ethylhexyl) sulfosuccinate (AOT) $(\mathrm{M}=444.6 \mathrm{~g} / \mathrm{mol})$ and polyoxyethylene (23) lauryl ether (Brij 35) $(\mathrm{M}=1199.5 \mathrm{~g} / \mathrm{mol})$ (Sigma-Aldrich) (purity $99 \%$ ) was used for agueous solution preparation. For preparation of aqueous solutions of surfactants doubly distilled and deionized water (Destamat $\mathrm{Bi} 18 \mathrm{E}$ ) was used. The surface tension of water $\left(\gamma_{L V}=72.8 \mathrm{mN} / \mathrm{m}\right)$ was always controlled at $20^{\circ} \mathrm{C}$ by Krüss $\mathrm{K} 9$ tensiometer under atmospheric pressure by the ring method before the solutions were prepared. For the contact angle measurements the AOT+Brij 35 mixtures at different mole fraction of surfactants in the bulk phase $(\alpha)$ were prepared. The polytetrafluoroethylene (PTFE) plates used for contact angle measurements were prepared from PTFE homopolymer, obtained as a result of free-radical polymerization of tetrafluoroethylene in the presence of peroxides as initiators by the Nitrogen Industrial Plant in Tarnów (Poland). The plate were cut from a large sheet of PTFE, mechanically polished and cleaned by triple boiling in $\mathrm{HCl}(1: 1)$ and triple boiling in doubly distilled and deionized water. Their final cleaning in doubly distilled water was performed in an ultrasonic bath for twenty minutes; the plates were then dried by blowing hot air. The quality of the surface of each plate was controlled by a polarizing microscope (Nikon, ECLIPSE E600 POL). Plates with a smooth surface characterized by a AFM techniques were used for contact angle measurements. 


\subsection{Contact angle measurements}

The advancing contact angle for water and aqueous solution mixtures of AOT+Brij 35 on PTFE plates was measured with sessile drop method by using the telescope-goniometer system at $25 \mathrm{x}$ magnification in a thermostated measuring chamber at $20 \pm 0.1^{\circ} \mathrm{C}$. After settling a drop of aqueous solution of the surfactant at a given concentration in the range from 0 to $10^{-2} \mathrm{M}$ on the PTFE plate the contact angle was read immediately on both sides of the drop. The measurements were repeated several times by settling other drops on the same plate. Next, a new plate was placed in the chamber and the above procedure was repeated. The reported contact angle values were the average of at least 10 independent drops.

\subsection{Surface tension measurements}

Surface tension measurements of the AOT and Brij 35 aqueous solutions were made by ring method using a Krüss K9 tensiometer, under the atmospheric pressure. In all cases, more than five successive measurements were carried out and the standard deviation did not exceed $\pm 0.2 \mathrm{mN} / \mathrm{m}$. The temperature was controlled within $\pm 0.1^{\circ} \mathrm{C}$.

\section{RESULTS AND DISCUSSION}

Surface tension, $\gamma_{L V}$, values of aqueous solutions of sodium bis(2ethylhexyl) sulfosuccinate (AOT) and polyoxyethylene (23) lauryl ether (Brij 35) and their mixtures are presented in Fig. 1 (curve 1-6), in the form of $\gamma_{L V}$ versus $\log C$ plots. $C$ is the total concentration of the surfactant in the bulk phase. A curve break, occurs at the critical micelle concentration (CMC), in which the surfactant aggregates forming micelles. From Fig. 1 it is seen, that the surface tension of the aqueous AOT + Brij 35 binary mixture solutions depends on the total concentration and the composition of the mixture. The CMC value increasing with the increase of the AOT quantity in the mixture (Fig. 1). Above the CMC the surface tension of the aqueous AOT + Brij 35 mixture solutions remains constant, but only in the case of the $0.2 \mathrm{AOT}+0.8$ Brij 35 mixture. 


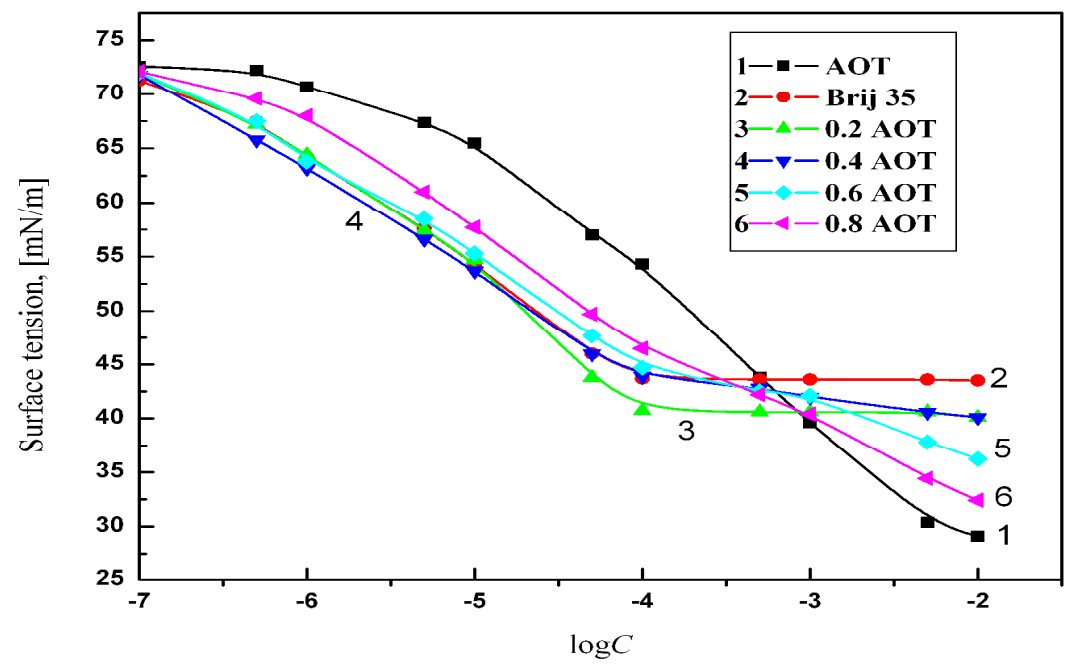

Fig. 1. A plot of the surface tension $\left(\gamma_{L V}\right)$ of the aqueous solutions of the AOT and Brij 35 and their mixtures vs. the AOT mole fraction in the solution $(\alpha)$. Curves 3, 4, 5 and 6 correspond to the mole fraction of AOT in the mixture equal to $0.2 ; 0.4 ; 0.6$ and 0.8 respectively.

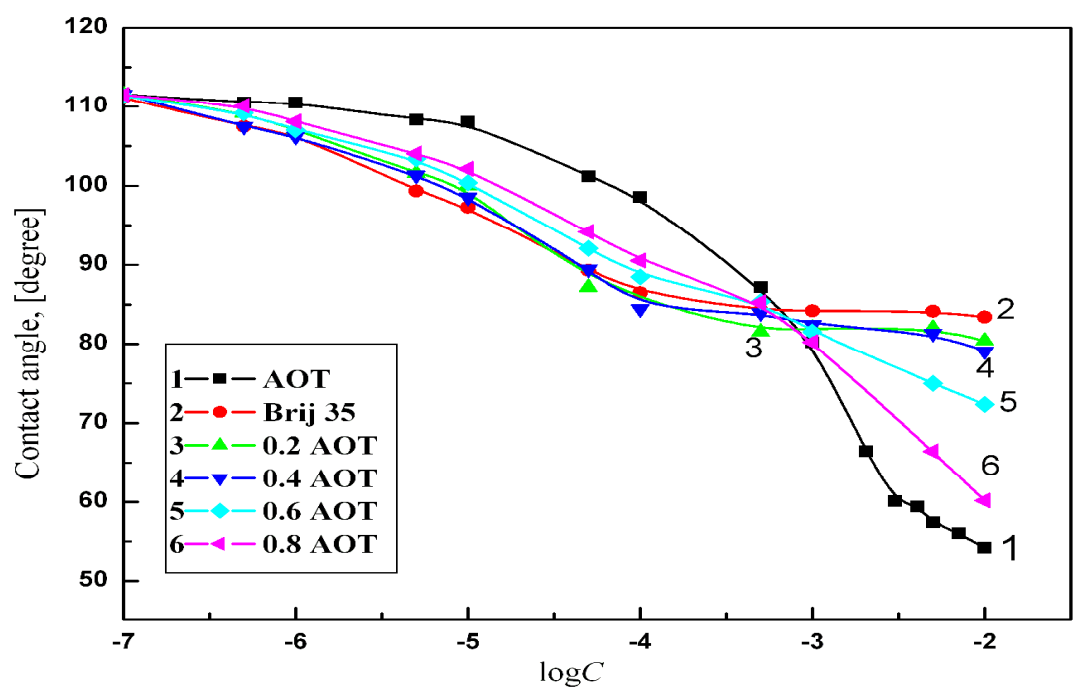

Fig. 2. A plot of the contact angle $(\theta)$ of the aqueous solutions of the AOT and Brij 35and their mixture on the PTFE surface vs. the logarithm of the surfactants total concentration $(\log C)$. Curves from 2 to 6 correspond to the mole fraction of AOT in the mixture equal to $0.2 ; 0.4 ; 0.6$ and 0.8 respectively. 
Adsorption of the surfactant at the water-air interface influences the contact angle of aqueous AOT, Brij 35 and their mixture solutions on the PTFE surface. The obtained results of the contact angle measurements are shown in Fig. 2 as a function of the logarithm of surfactant total concentration. From this figure it is seen, that in the $\log C$ range from -7 to -6 the contact angle values are slightly changed. At the surfactant concentration close and higher than $10^{-5} \mathrm{M}$ a considerable decrease of the contact angle of the solutions at the PTFE surface is seen. Of course the biggest changes of the contact angle occurring at the surfactant concentration range corresponding to the saturated monolayer of surfactant at the water-air interface. From the Fig. 2 it is also seen, that PTFE wetting strongly depends on the total concentration of the surfactant and the composition of the solution. For the better analysis of the changes of the contact angle with the change of the surfactants mixture composition the obtained values of the contact angle on the PTFE surface were presented as a function of the mole fraction of AOT in the bulk phase $(\alpha)$ of the mixtures solution (Fig. 3).

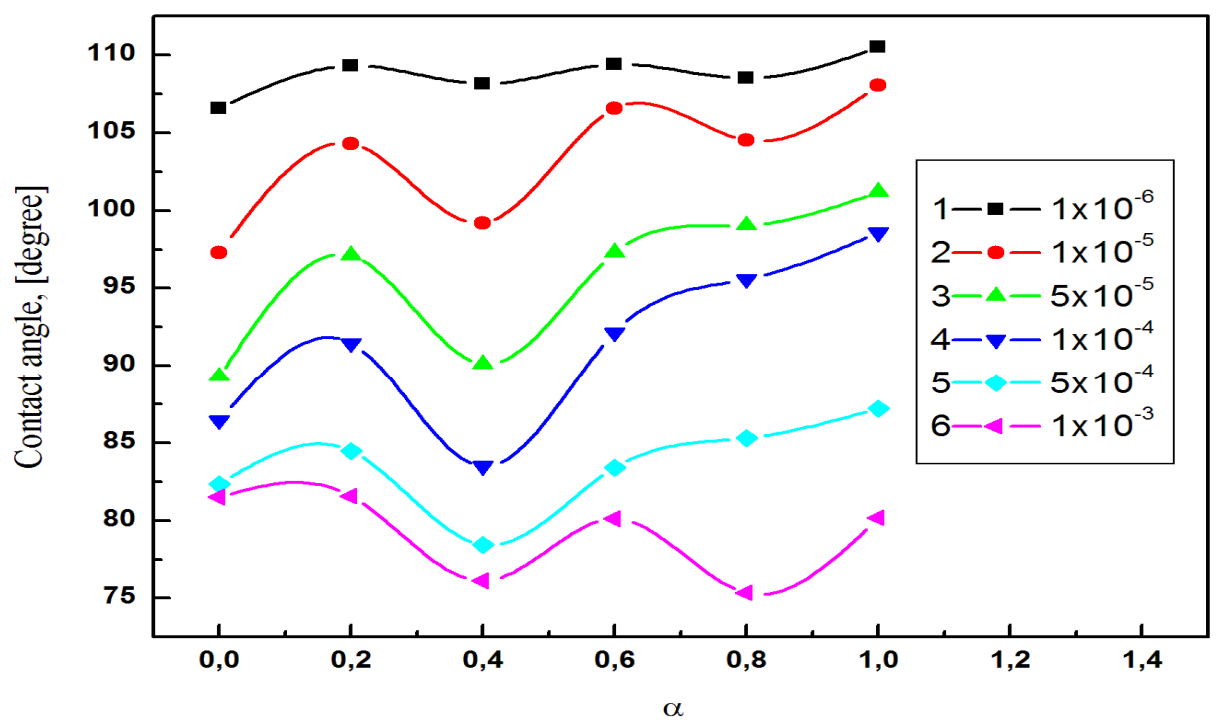

Fig. 3. A plot of the contact angle $(\theta)$ of the aqueous solutions of the AOT and Brij 35 and their mixture on the PTFE surface vs. the mole fraction of AOT in the mixture $(\alpha)$ for the total surfactants concentration equal to: $1 \cdot 10^{-6} \mathrm{M}, 1 \cdot 10^{-5} \mathrm{M}, 5 \cdot 10^{-5} \mathrm{M}, 1 \cdot 10^{-4} \mathrm{M}, 5 \cdot 10^{-4} \mathrm{M}$ and $1 \cdot 10^{-3} \mathrm{M}$. 
From this figure it is seen that for the 0.4 AOT + 0.6 Brij 35 mixture there is a negative deviation of the values of the contact angle and it can be connected with the synergism between the AOT and Brij 35 in the reduction of the contact angle of water on the PTFE surface.

Zisman [15] characterized wetting of hydrophobic surface (for example PTFE) by measuring the contact angle for different liquids and plotting $\cos \theta$ vs. $\gamma_{L V}$. Extrapolation of such plots to $\cos \theta=1$ allows estimation of $\gamma_{C}$, that is so-called critical surface tension of wetting. However, in the studied systems (AOT + Brij 35) there is no linear dependence between the $\cos \theta$ and $\gamma_{L V}$ (Fig. 4).

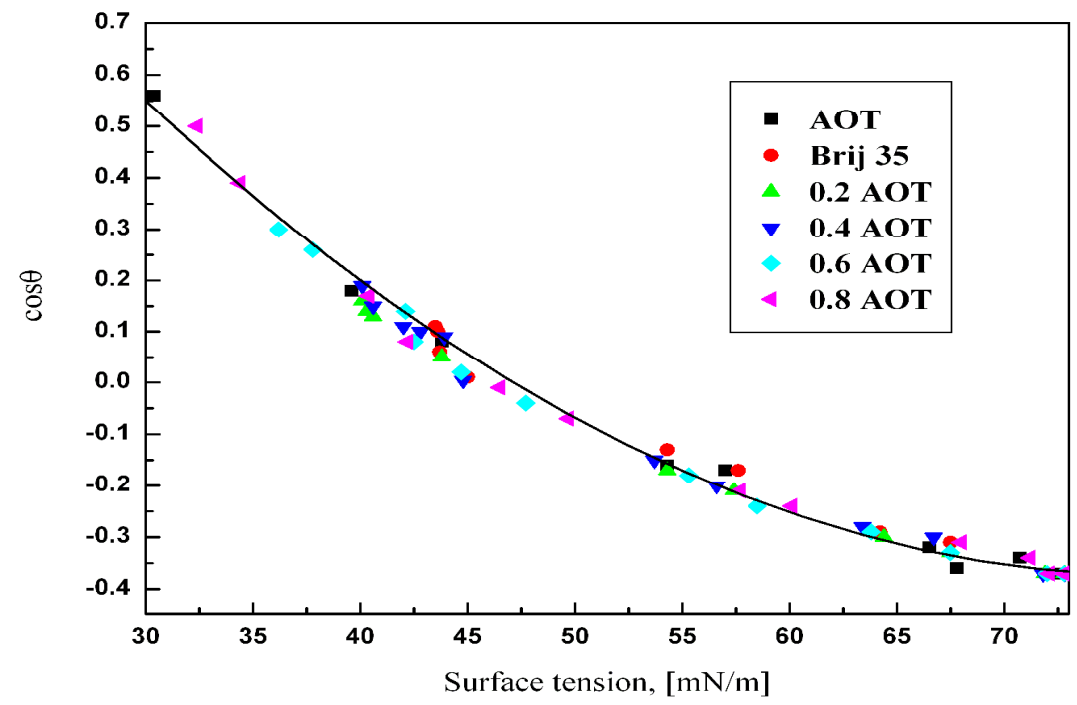

Fig. 4. A plot of the cosine of the contact angle $(\theta)$ of the aqueous solutions of the AOT and Brij 35and their mixtures on the PTFE surface vs. the surface tension of the solution.

Thus, according to the Bargeman and van Voorst Vader suggestion the relationship between the adhesion tension $\left(\gamma_{L V} \cos \theta\right)$ and surface tension were determined and presented on the Fig. 5. From this figure it is seen, that really there is a linear dependence between the adhesion and surface tension of aqueous solutions of AOT, Brij 35 and their mixtures, which is expressed by the following equation:

$$
\gamma_{L V} \cos \theta=-\gamma_{L V}+48.3
$$


On the basis of the Eq. (3) the critical surface tension of the PTFE wetting were determined and it is equal to $24.2 \mathrm{mN} / \mathrm{m}$. This value is somewhat higher than that determined from the contact angles of $\mathrm{n}$-alkanes on the PTFE surface $(20.2 \mathrm{mN} / \mathrm{m})$, and does not depend on the kind of the surfactant and the composition of the solution.

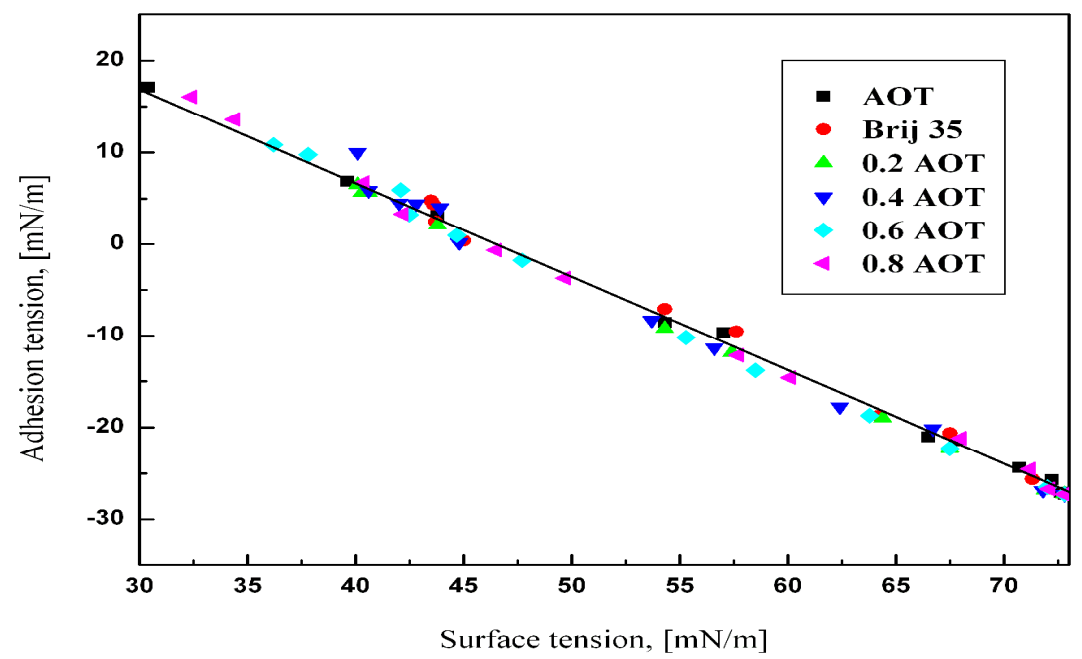

Fig. 5. A plot of the adhesion tension of the aqueous solutions of the AOT and Brij 35and their mixtures to the PTFE surface vs. the surface tension of the solution.

Wetting of solids depends not only on the surface tension of the liquid, but also on the solid-liquid interfacial tension [2].Knowing the surface tension of PTFE and assuming, that it does not change after settling a drop of the studied solutions on it, we can determine the PTFEsolution interfacial tension $\left(\gamma_{S L}\right)$ from the Young equation [2]. Substituting into Eq. (1), $\gamma_{S V}=20.2 \mathrm{mN} / \mathrm{m}$ and values from Fig. 5 (curve 1-6), the values of $\gamma_{S L}$ were calculated and presented in Fig. 6 as a relationship between the $\gamma_{S L}$ and $\log C$. From this figure it is seen, that the PTFE-solution interfacial tension depends on the total surfactant concentration and on the composition of the solution, similarly as the solution surface tension (Fig.1). 


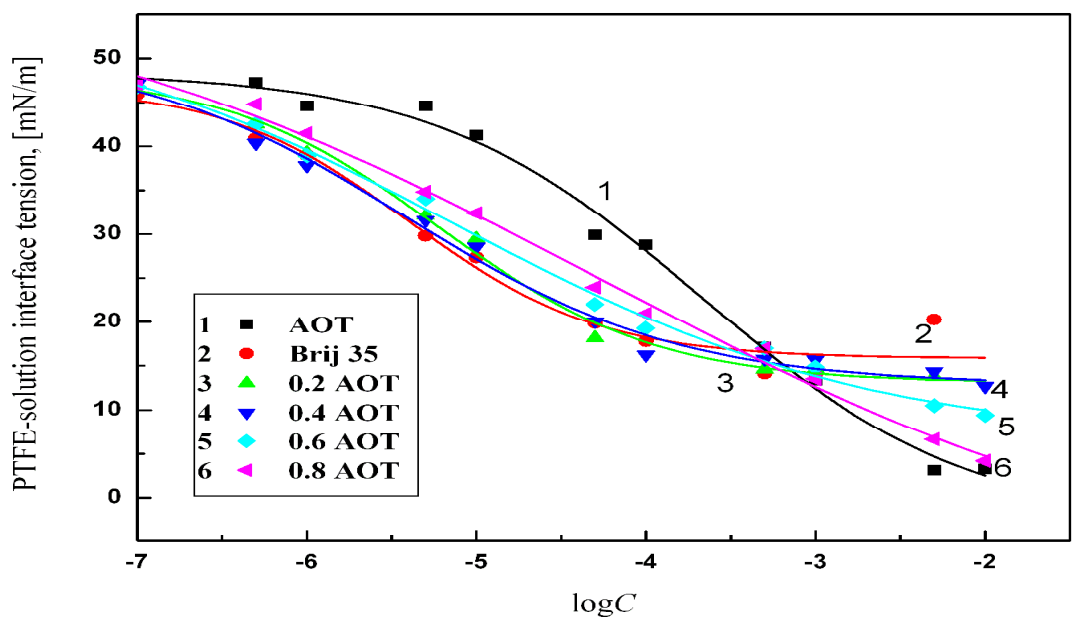

Fig. 6. A plot of the adhesion tension of the aqueous solutions of the AOT and Brij 35and their mixtures to the PTFE surface vs. the surface tension of the solution.

\section{CONCLUSIONS}

1. Wettability of the PTFE surface strongly depends on the concentration of the surfactant in the solution and its composition.

2. The biggest changes of the contact angle of the aqueous solutions of the studied surfactants and their mixtures on the PTFE surface correspond to the surfactants concentration range refering to the saturated monolayer at the water-air interface.

3. For the $0.4 \mathrm{AOT}+0.6 \mathrm{Brij} 35$ mixture there is a negative deviation of the values of the contact angle and it can be connected with the synergism between the AOT and Brij 35 in the reduction of the contact angle of water on the PTFE surface.

4. There is a linear dependence between the adhesion and surface tension of aqueous solutions of AOT, Brij 35 and their mixtures.

5. The PTFE-solution interfacial tension depends on the total surfactant concentration and on the composition of the solution, similarly as the solution surface tension. 


\section{REFERENCES}

[1] B. Jańczuk, W. Wójcik and A. Zdziennicka, Mater. Chem. Phys., 58, 166, (1999).

[2] J. M. Rosen, "Surfactants and Interfacial Phenomena", Wiley Interscience, New York, Chapter 6, 1989.

[3] B. Jańczuk, W. Wójcik and A. Zdziennicka, Eur. Polym. J., 33, 1093, (1997).

[4] B. Jańczuk, M. L. González and J. M. Bruque, Canadian Metal. Quart., 35, 17, (1996).

[5] A. Zdziennicka, B. Jańczuk and W. Wójcik, J. Colloid Interface Sci., 268, 200, (2003).

[6] L. Hołysz and E. Chibowski, Langmuir, 8, 303, (1992).

[7] C. S. Gau and G. Zografi, J. Colloid Interface Sci., 140, 1, (1990).

[8] A. Mavon, H. Zahouani, D. Redoules, P. Agache, Y. Gall and P. Humbert, Colloids and Surfaces B:Biointerfaces, 8,147, 1997.

[9] A. Mavon, D. Redoules, P. Humbert, P. Agache and Y. Gall, Surfaces B:Biointerfaces, 10, 243, 1998.

[10] P. G. Agache, P. Humbert (Eds.) and H. J. Maibach, "Measuring the skin", Springer, 2004.

[11] A. W. Adamson, "Physical Chemistry of Surfaces", $5^{\text {th }}$ Ed., WileyInterscience, New York, 1991.

[12] E. H. Lucassen-Reynders, J. Phys. Chem., 67, 969 (1963).

[13] M. K. Bernett and W. A. Zisman, J. Phys. Chem., 63, 1241, (1959).

[14] M. K. Bernett and W. A. Zisman, J. Phys. Chem., 63, 1911 (1959).

[15] W. A. Zisman, "Advances in Chemistry Series", vol. 43, American Chemical Society, Washington, DC, p.1, 1964.

[16] D. Bargeman and F. Van Voorst Vader, J. Colloid Interface Sci., 42, 467, (1973).

[17] R. A. Pyter, G. Zografi and P. Mukerjee, J. Colloid Interface Sci., 89, 144 (1982).

[18] R. J. Good, J. Adhesion Sci. Technol., 6 (12), 1269, (1992).

[19] T. D. Blake, "Wetting", in: Th.F. Tadros (Ed.), "Surfactants", Academic Press, London, 1984.

[20] Y. Kitazaki and T. Hata, J. Adhesion, 4, 123, (1972).

[21] Y. Kitazaki, T. Hata and L. H. Lee (Ed.), "Recent Advances in Adhesion", Gordon and Breach, New York, 1973. 


\section{CURRICULUM VITAE}

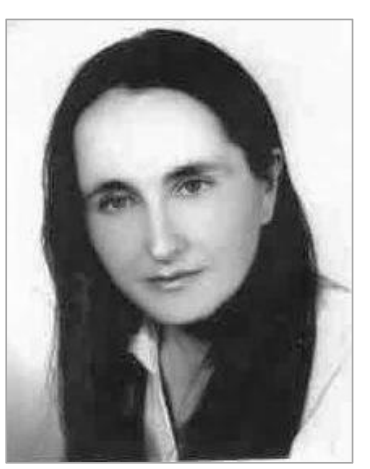

Joanna Krawczyk. Graduated from the Faculty of Chemistry, Maria Curie-Sklodowska Univer-sity in 2004 r. In 2009 r. she obtained Ph.D. degree and since $2010 \mathrm{r}$. has been held a position of adjunct in the Department of Interfacial Phenomena, Faculty of Chemistry, Maria Cure-Sklodowska University. She is an author of 16 papers. Main fields of a scientific interest are physico-chemical properties of surfactants and interfacial phenomena in the systems including surface active agents, with a special regard to the natural surfactants. 